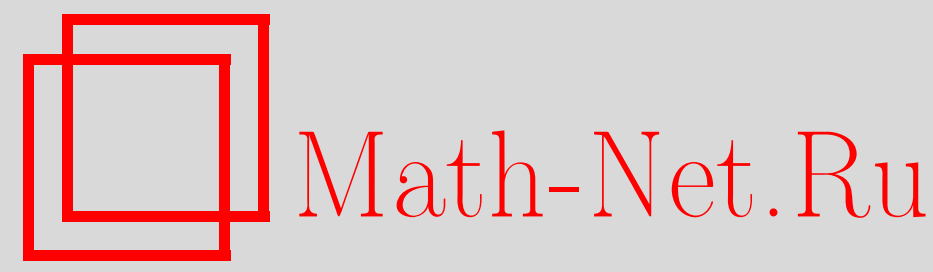

И. Х. Сабитов, Изометрические преобразования поверхности, порождающие конформные отображения ее на себя, Матем. сб., 1998, том 189, номер 1, 119-132

DOI: https://doi.org/10.4213/sm297

Использование Общероссийского математического портала Math-Net.Ru подразумевает, что вы прочитали и согласны с пользовательским соглашением http://www . mathnet.ru/rus/agreement

Параметры загрузки:

IP: 3.91 .87 .62

26 апреля 2023 г., $15: 30: 16$ 
УДК 513.7

\author{
И.Х. Сабитов
}

\title{
Изометрические преобразования поверхности, порождающие конформные отображения ее на себя
}

\begin{abstract}
Рассматриваются изометрии поверхности, порождающие на ней по определенной схеме конформные отображения на себя. Дается полное описание поверхностей, допускающих изометрии, которым соответствуют тождественные конформные отображения. Для общего случая приводится рядрезультатов, а также формулируются нерешенные вопросы.

Библиографиия: 8 названий.
\end{abstract}

1. В работе [1] мы показали, что изгибания или достаточно малые изометрические преобразования поверхности порождают на поверхности квазиконформные отображения ее на себя (в поря дке напоминания описание происхождения этих квазиконформных отображений дано в конце статьи в п. 8), и там же изучили случай, когда эти квазиконформные отображения оказьваются конформньми в силу предположения, что поверхность изгибается со скольжением по самой себе. В этой работе мы предположим априори, что порожденные по схеме из [1] квазиконформные отображения являются конформньми, и выясним, какие поверхности допускают изометрические деформации, которым соответствуют тождественные конформные отображения, и какова природа этих деф̆ормаций. Кроме того, в п. 8 мы обсудим некоторые общие вопросы, относящиеся к постановке задачи с конформными отображениями, и приведем некоторые конкретные результаты.

2. Пусть $S: \mathbf{r}=\mathbf{r}(u, v)$ - данная поверхность класса гладкости $C^{2}$,

$$
S^{*}: \mathbf{r}^{*}(u, v)=\mathbf{r}(u, v)-h(u, v) \mathbf{n}(u, v)
$$

- изометричная ей поверхность класса гладкости $C^{1}$, где $\mathbf{n}(u, v)$ - единичная нормаль к $S$. Изометрия между $S$ и $S^{*}$ предполагается по равенству внутренних координат. Это означает, что нормальный к $S$ вектор $-h \mathbf{n}$ "приходит" из точки $M \in S$ с радиус-вектором $\mathbf{r}(u, v)$ в точку $M^{*} \in S^{*}$ с радиус-вектором $\mathbf{r}^{*}(u, v)$, соответствуюшую $M$ по изометрии. Иначе говоря, мы сейчас рассматриваем случай, в некотором смысле противоположный к изгибаниям скольжения - на этот раз изометрия дается деформацией поверхности по нормали. В этом случае порожденное изометрической деформацией квазиконформное отображение поверхности на себя является тождественным отображением.

Работа выполнена при финансовой поддержке Петербургского конкурсного центра по фундаментальному естествознанию (грант № 95-0-1.3-103).

(С) И.Х. САвитов 1998 
Tеорема 1. Пусть $S: \mathbf{r}=\mathbf{r}(u, v) \in C^{2} u S^{*}: \mathbf{r}^{*}=\mathbf{r}(u, v)-h(u, v) \mathbf{n}(u, v) \in C^{1}$ - изометричные поверхности с изометрией по равенству внутренних координат $(u, v)$. Тогда $S$ допускает описанную выше изометрию "по нормали" только в следующих случаях:

1) $S$ - сфера или область на сфере;

2) $S$ - параболоид вращения или область на нем;

3) $S$ - плоскость или плоская область;

4) $S$ - иилиндрическая поверхность.

$B$ первых трех случаях изометрия $S \rightarrow S^{*}$ тривиальная, а в четвертом случае есть как тривиальные, так и нетривиальные изометрии, для которых сдвиг $h$ по нормали определяется решением некоторого обыкновенного дифференциального уравнения.

Заметим, мы вовсе не предполагаем, что $S$ априори является компактной или полной поверхностью, так что теорема утверждает, что нетривиальных изометрий “по нормали” нет и “в малом” (кроме случаев цилиндрической поверхности), причем от изометричной поверхности $S^{*}$ требуется предельно малая гладкость класса $C^{1}$. От самой поверхности $S$ требуется минимальная классическая гладкость $C^{2}$.

Доказательство теоремы мы дадим в пп. 3-7, исключая п. 4, посвященный усилению одного вспомогательного утверждения, встречающегося в самом доказательстве как некоторый момент в ходе рассуждений.

3. Выведем сначала условия на функцию $h(u, v)$, при которых поверхности $S: \mathbf{r}=\mathbf{r}(u, v) \in C^{2}$ и $S^{*}: \mathbf{r}^{*}=\mathbf{r}(u, v)-h(u, v) \mathbf{n}(u, v) \in C^{1}$ имеют одинаковую метрическую форму. Так как $h(u, v)=\mathbf{r}(u, v) \mathbf{n}(u, v)-\mathbf{r}^{*}(u, v) \mathbf{n}(u, v)$, то $h(u, v) \in C^{1}$, и поэтому можем вычислять $d s^{2}$ и $d s^{*^{2}}$ по обычньм формулам. Дальнейшие вычисления сильно упрошаются, если мы перейдем к изотермическим координатам $(\xi, \eta)$, в которых $d s^{2}=\lambda^{2}(\xi, \eta)\left(d \xi^{2}+d \eta^{2}\right)$. В этих координатах метрика имеет гладкость класса $C^{1, \alpha}$ с любым $\alpha<1$, поэтому переход от координат $(u, v)$ к $(\xi, \eta)$ будет класса гладкости $C^{2}$ (см. [2]), так что гладкость радиус-вектора поверхности не изменится. Вычисляя метрическую форму $d s^{*^{2}}$ поверхности $S^{*}$ и приравнивая ее к $d s^{2}(\xi, \eta)$, получаем три уравнения (в которых использованы стандартные обозначения коэффициентов второй квадратичной формы, средней и гауссовой кривизн):

$$
\begin{aligned}
2 h L+\left(2 H L-K \lambda^{2}\right) h^{2}+h_{\xi}^{2} & =0, \\
2 h M+2 H M h^{2}+h_{\xi} h_{\eta} & =0, \\
2 h N+\left(2 H N-K \lambda^{2}\right) h^{2}+h_{\eta}^{2} & =0 .
\end{aligned}
$$

Исключая из этих уравнений $h_{\xi}$ и $h_{\eta}$, получаем уравнение для $h$ :

$$
K h\left(4+4 H h+K h^{2}\right)=0 .
$$

Считаем сначала, что $K \neq 0$. Тогда из уравнения (3) находим $h$ :

$$
h=-2 R_{1} \text { или } h=-2 R_{2} \text {, }
$$


где $R_{1}$ и $R_{2}$ - главные радиусы кривизны поверхности $S$. Следовательно, мы видим, что при $K \neq 0$ изгибаний "по нормали" не бывает. Выразим $h$ через один из главных радиусов кривизны, обозначим его через $R$, и подставив в (2), получим для него уравнения (с учетом того, что $2 H=1 / R+K R$ ):

$$
\begin{gathered}
R_{\xi}^{2}=K R^{2}\left(\lambda^{2}-R L\right), \\
R_{\eta}^{2}=K R^{2}\left(\lambda^{2}-R N\right), \\
R_{\xi} R_{\eta}=-K M R^{3} .
\end{gathered}
$$

Сложив первые два уравнения в (4), имеем $R_{\xi}^{2}+R_{\eta}^{2}=K R^{2} \lambda^{2}\left(1-K R^{2}\right)$, откуда видно, что случай $K<0$ невозможен. Тем самым, остается только возможность $K>0$; кроме того, отсюда же получаем, что $R$ должен быть минимальньм (по модулю) из главных радиусов кривизны. Если на поверхности всюду $R_{\xi}=R_{\eta}=0$, то поверхность будет состоять из омбилических точек, поэтому будет сферой (или областью на сфере). Пусть есть точка, в которой $\operatorname{grad} R \neq 0$. Рассмотрим окрестность этой точки.

Из уравнений (4) получаем, что $-M R_{\xi}^{2}+(L-N) R_{\xi} R_{\eta}+M R_{\eta}^{2}=0$, т.е. направления $\left\{R_{\xi}, R_{\eta}\right\}$ и $\left\{-R_{\eta}, R_{\xi}\right\}$ являются главными направлениями. Первое из этих направлений соответствует $\operatorname{grad} R$ на плоскости переменных $(\xi, \eta)$, а второе направлению линий уровня $R=$ Const. Легко проверить, что $1 / R$ является главной кривизной вдоль второго главного направления. Следовательно, мы пришли к важному наблюдению: линии кривизны одного из семейств определяются как линии уровня соответствующей главной кривизны. Все дальнейшие рассуждения будут основаны на использовании этого факта.

Пусть $\sigma$ - какая-либо линия кривизны из указанного семейства, $\mathbf{r}=\mathbf{r}(s)-$ eе уравнение. Рассмотрим развертьвающуюся поверхность с направляющей $\sigma$ и образуюшими вдоль нормалей. Горловая линия этой поверхности вырождается в точку, так как ее радиус-вектор $\mathbf{r}(s)+R \mathbf{n}$ имеет производную, равную нулю; поэтому соответствуюшая развертываюшаяся поверхность является конусом, а сама линия $\sigma$ является сферической. Пусть кривая $\gamma$ с радиус-вектором $\mathbf{r}=\mathbf{r}(\beta)$ - произвольная линия кривизны другого семейства, которую пересекают линии кривизны семейства $R=\beta$. Тогда концевые точки векторов $R \mathbf{n}$ вдоль $\gamma$ образуют некоторую гладкую кривую $\Gamma$ с радиус-вектором $\mathbf{l}(\beta)=\mathbf{r}(\beta)+R(\beta) \mathbf{n}(\beta)$, причем кривая Г от выбора линии кривизны $\gamma$ не зависит.

Пусть $R(\xi, \eta)=\beta$ - гладкое (по $\beta$ ) семейство гладких линий $\sigma_{\beta}$ на плоскости $(\xi, \eta)$, соответствуюших линиям кривизны, которые имеют уравнения вида $\xi=\xi(\eta, \beta) ;$ пусть $\eta=\eta(\xi)+\alpha-$ трансверсальное к предыдушему семейство гладких линий, причем пусть значению $\alpha=0$ соответствует линия кривизны $\gamma$. Нетрудно проверить, что в окрестности рассматриваемой точки существует гладкий переход $(\xi, \eta) \rightarrow(\alpha, \beta)$, поэтому поверхность $S$ можно представить в виде $\mathbf{r}(\alpha, \beta)=\mathbf{l}(\beta)-\beta \mathbf{n}(\alpha, \beta)$. В рассматриваемой точке линии $\beta=\beta_{0}$ и $\alpha=0$ пересекаются как линии кривизны, поэтому получаем, что в этой точке поверхности векторы $\mathbf{r}_{\beta}^{\prime}$ и $\mathbf{r}_{\alpha}^{\prime}$ ортогональны. Значит, в ней же ортогональны векторы $\mathbf{l}_{\beta}^{\prime}$ и $\mathbf{r}_{\alpha}^{\prime}$ (так как в этой точке $\mathbf{l}_{\beta}^{\prime}=\mathbf{r}_{\beta}^{\prime}+\mathbf{n}+\beta \mathbf{n}_{\beta}^{\prime}=\mathbf{r}_{\beta}^{\prime}(1-\beta k)+\mathbf{n}$, где $k$ - вторая главная кривизна). Заметим, что это рассуждение применимо в любой точке кривой $\sigma_{\beta_{0}}$, надо только базовую линию кривизны вида $\eta=\eta(\xi)$ выпускать 
из этой точки, а линия Г при этом останется без изменения. Следовательно, вектор $\mathbf{l}_{\beta}^{\prime}$ ортогонален касательной к линии кривизны $\sigma$ в каждой ее точке, а так как вектор $\mathbf{l}_{\beta}^{\prime}$ постоянен вдоль $\sigma$, то линия $\sigma$ - плоская. Кроме того, как мы установили раньше, она является сферической, следовательно, $\sigma$ - дуга окружности. Такие же рассуждения применимы к любой линии кривизны семейства с $R=$ Const, поэтому все они в окрестности рассматриваемой точки являются дугами окружностей. Так как главная кривизна поверхности вдоль $\sigma$ постоянна, а кривая $\sigma$ - окружность, то угол между нормалью к $S$ вдоль $\sigma$ и плоскостью окружности $\sigma$ постоянен. Получаем, что поверхность $S$ устроена или как каналовая поверхность, или как поверхность вращения, и чтобы установить точное ее строение, нужно выяснить, расположены ли центры дуг окружностей вида $\sigma$ вдоль одной прямой или нет.

Прежде чем устанавливать точньй вид поверхности $S$, посмотрим, какие поверхности врашения допускают изометрические преобразования исследуемого вида. Покажем, что таких поверхностей вращения всего три: параболоид вращения, сфера и круговой иилиндр.

Действительно, пусть локально $S$ имеет стандартное уравнение $\mathbf{r}=u \mathbf{k}+$ $\psi(u) \mathbf{e}(v)$, где $\mathbf{e}=\mathbf{i} \cos v+\mathbf{j} \sin v$. Тогда нетрудно вычислить, что поверхность $S^{*}$ имеет радиус-вектор $r^{*}=\left(u+2 \psi \psi^{\prime}\right) \mathbf{k}-\psi \mathbf{e}$. Из условия изометричности $S$ и $S^{*}$ получаем, что меридиан $\psi=\psi(u)$ должен удовлетворять уравнению $\left(1+2 \psi^{\prime 2}+2 \psi \psi^{\prime \prime}\right)^{2}=1$, откуда имеем две возможности: 1$) \psi^{\prime 2}+\psi \psi^{\prime \prime}=0$, т.е. $\psi^{2}=u C_{1}+C_{2}$, или же 2) $1+\psi^{\prime 2}+\psi \psi^{\prime \prime}=0$, т.е. $\psi^{2}+\left(u-C_{1}\right)^{2}=C_{2}^{2}$. В первом случае при $C_{1} \neq 0$ получаем, что поверхность $S$ должна быть параболоидом вращения (или областью на нем), а при $C_{1}=0$ - цилиндром вращения (или областью на нем). Во втором случае $S$ является сферой (или областью на ней). Тем самьм высказанное утверждение доказано. Заметим, что для сферы изометрия $S \rightarrow S^{*}$ сводится к симметрии относительно центра сферы, в случае параболоида - к сдвигу параболоида вдоль оси врашения с последующим поворотом вокруг оси на $180^{\circ}$, а в случае цилиндра - к симметрии относительно оси цилиндра.

Вернемся к рассмотрению общего случая. Временно предположим, что поверхность $S$ имеет исходную гладкость класса $C^{3}$, тогда на ней в окрестности рассматриваемой неомбилической точки можно ввести координаты в линиях кривизны (в классе $C^{2}$ для дифференциальных уравнений линий кривизны есть только теорема существования, но нет теоремы единственности, поэтому возможность введения их как координатных линий остается необоснованной; примеры подтверждают [3], что, например, в случае асимптотических линий это действительно возможно не всегда, и хотя для линий кривизны такие примеры нам неизвестны, но нам неизвестно и обоснование такой возможности), причем переход от $(\xi, \eta)$ к координатам $(\alpha, \beta)$ в линиях кривизны будет класса $C^{1}$. Тогда уравнения поверхностей $S$ и $S^{*}$ можно представить соответственно в виде

$$
\begin{aligned}
\mathbf{r}(\alpha, \beta) & =\mathbf{l}(\beta)-R(\beta) \mathbf{n}(\alpha, \beta), \\
\mathbf{r}^{*}(\alpha, \beta) & =\mathbf{l}(\beta)+R(\beta) \mathbf{n}(\alpha, \beta) .
\end{aligned}
$$

Из условия совпадения метрик $S$ и $S^{*}$ получаем следующие уравнения

$$
\begin{gathered}
\left(\mathbf{l}^{\prime} \mathbf{n}_{\alpha}^{\prime}\right)=0 \text { или }\left(\mathbf{l}^{\prime} \mathbf{r}_{\alpha}^{\prime}\right)=0, \mathbf{l}^{\prime}=\mathbf{l}^{\prime}(\beta), \\
R^{\prime}(\beta)\left(\mathbf{n} \mathbf{l}^{\prime}\right)-k R\left(\mathbf{l}^{\prime} \mathbf{r}_{\beta}^{\prime}\right)=0,
\end{gathered}
$$

где $k$ - главная кривизна в направлении $\beta$-линии. 
С другой стороны, из (5) имеем

$$
(1-k R) \mathbf{r}_{\beta}^{\prime}=\mathbf{l}^{\prime}(\beta)-R^{\prime}(\beta) \mathbf{n},
$$

откуда получаем, что $\left(\mathbf{n l}^{\prime}(\beta)\right)=R^{\prime}(\beta)$ и $(1-k R)\left(\mathbf{r}_{\beta}^{\prime} \mathbf{l}^{\prime}(\beta)\right)=\mathbf{l}^{\prime 2}-R^{\prime 2}$. Подставляя найденные значения $\left(\mathbf{n} \mathbf{l}^{\prime}(\beta)\right)$ и $\left(\mathbf{r}_{\beta}^{\prime} \mathbf{l}^{\prime}(\beta)\right)$ в $(5)$, получаем $R^{\prime 2}(\beta)=k R \mathbf{l}^{\prime 2}(\beta)$, т.е. вторая главная кривизна $k$ тоже зависит только от $\beta$.

Так как правая часть уравнения (7) имеет производную по $\alpha$, то есть производная и левой части. Продифференцируем (7) по $\alpha$. Учитывая, что вдоль $\alpha$-линии имеем $\mathbf{n}_{\alpha}^{\prime}=-(1 / R) \mathbf{r}_{\alpha}^{\prime}$, получаем уравнение

$$
(1-k R) \mathbf{r}_{\beta \alpha}^{\prime \prime}=\left(R^{\prime} / R\right) \mathbf{r}_{\alpha}^{\prime}
$$

На (8) можно смотреть как на обыкновенное линейное дифференциальное уравнение относительно $\mathbf{r}_{\alpha}^{\prime}$. Решая его, находим, что $\mathbf{r}_{\alpha}^{\prime}$ имеет вид

$$
\mathbf{r}_{\alpha}^{\prime}=f(\beta) \mathbf{F}(\alpha)
$$

где скалярная функция $f(\beta)$ равна $\exp \left(\int(1-k R)^{-1}\left(R / R^{\prime}\right) d \beta\right)$. Так как в линиях кривизны $\left(\mathbf{r}_{\alpha}^{\prime} \mathbf{r}_{\beta}^{\prime}\right)=0$, то из $(7)$ и $(9)$ получаем, что $\left(\mathbf{F}(\alpha) \mathbf{l}^{\prime}(\beta)\right)=0$, следовательно, вектор $\mathbf{l}^{\prime}(\beta)$ имеет постоянное направление (так как векторы $\mathbf{F}(\alpha)$ заведомо не параллельны хотя бы при двух разных $\alpha)$.

Покажем теперь, что поверхность $S$ является поверхностью вращения. Пусть окружности (или дуги окружностей) $\sigma$ на $S$ имеют центры с радиус-вектором $\mathbf{p}(\beta)$; нормали $\mathbf{n}$ к $S$ вдоль $\sigma$ сходятся в точке с радиус-вектором $\mathbf{l}(\beta)$. Рассмотрим на $\sigma$ произвольную точку с радиус-вектором $\mathbf{r}(\alpha, \beta)$. Так как в треугольнике с вершинами $\mathbf{r}(\alpha, \beta), \mathbf{l}(\beta), \mathbf{p}(\beta)$ стороны постоянны (при изменении $\alpha$ и фиксированном $\beta$ ), то вектор $\mathbf{p}(\beta)-\mathbf{l}(\beta)$ должен быть ортогонален к плоскости окружности $\sigma$. Выше мы установили, что $\mathbf{l}^{\prime}(\beta)$ ортогонален к плоскости окружности $\sigma$, следовательно, касательная к $\mathbf{l}(\beta)$ проходит через центр окружности $\sigma$. И так как $\mathbf{l}^{\prime}(\beta)$ имеет постоянное направление и линия действия этого вектора проходит через центр окружности $\sigma$, то получаем, что все окружности $\sigma$ имеют центры $\mathbf{p}(\beta)$, расположенные на одной прямой, идущей в направлении вектора $\mathbf{l}^{\prime}(\beta)$, ортогонального к плоскости каждой окружности $\sigma$, т.е. $S$ является поверхностью вращения. Следовательно, допустимая поверхность $S$ в рассматриваемом случае - когда кривизна больше нуля и точка, в окрестности которой ведется рассуждение, неомбилическая - единственная: параболоид врашения, причем, если $S$ имеет уравнение $\mathbf{r}=u \mathbf{k}+\sqrt{u C_{1}+C_{2}} \mathbf{e}(v)$, где $\mathbf{e}=\mathbf{i} \cos v+\mathbf{j} \sin v$, то $\mathbf{r}^{*}=\left(u+C_{1}\right) \mathbf{k}-\sqrt{u C_{1}+C_{2}} \mathbf{e}(v)$, так что $S^{*}$ получается из $S$ путем сдвига вдоль оси вращения на $C_{1}$ с последующим поворотом на $180^{\circ}$.

Если же предположить, что все рассматриваемые точки омбилические, то $S$ будет сферической поверхностью, а для нее, как было установлено вьшше, изометрия $S \rightarrow S^{*}$ тоже существует и сводится она к симметрии относительно центра сферы. 
4. В этом пункте мы отвлечемся от продолжения доказательства теоремы и покажем как полезный факт, что только что установленное утверждение о строении поверхности $S$ имеет место в более общей ситуации. Из данных в (7) и (9) выражений для $\mathbf{r}_{\beta}^{\prime}$ и $\mathbf{r}_{\alpha}^{\prime}$ получаем, что первая форма поверхности $S$ имеет вид $d s^{2}=f^{2}(\beta)|\mathbf{F}(\alpha)|^{2} d \alpha^{2}+g^{2}(\beta) d \beta^{2}$, где $g^{2}(\beta)=\left|\mathbf{r}_{\beta}^{\prime}\right|^{2}=\left(\mathbf{l}^{\prime 2}-R^{\prime 2}\right) /(1-k R)^{2}$, т.е. метрика поверхности является метрикой вращения. Для получения утверждения о том, что поверхность $S$ является поверхностью вращения, мы использовали дополнительную информацию о ее свойствах, связанную со свойствами нормалей к $S$ вдоль дуг окружностей $\sigma$ как линий кривизны. Между тем можно показать, что если метрика врашения реализована в $\mathbb{R}^{3}$ в виде некоторой поверхности $\Sigma$, расслоенной на окружности с центрами вдоль некоторой линии, то эта линия на самом деле является прямой и плоскости окружностей ортогональны к ней, так что $\Sigma$ является поверхностью вращения (т.е. тем самым утверждается локальная однозначная определенность поверхностей вращения в классе поверхностей с плоскими круговыми сечениями, соответствующими параллелям поверхности вращения, - с точностью до выбора кратности обхода параллелей). Точная формулировка следующая.

УтвеРЖДЕнИЕ. Пусть метрика $d s^{2}=d \rho^{2}+B^{2}(\rho) d \varphi^{2}, 0<\rho_{1}<\rho<\rho_{2}$, $-\varepsilon<\varphi<\varepsilon$, с непрерывным коэффичиентом $B(\rho)>0$, реализована в $\mathbb{R}^{3}$ в виде $C^{1}$-гладкой поверхности $\Sigma: \mathbf{r}(\rho, \varphi)=\mathbf{p}(\rho)+f(\rho) \mathbf{e}(\rho, \varphi)$, әде $\mathbf{p}(\rho), f(\rho), \mathbf{e}(\rho, \varphi) \in$ $C^{1}, \mathbf{e}^{2}=1$, и векторы $\mathbf{e}(\rho, \varphi)$ при фиксированных $\rho$ и переменных $\varphi$ лехат на плоскостях $\Pi_{\rho}$ с гладко изменяющейся единичной нормалью $\mathbf{q}(\rho)$ (т.е. линиям $\rho=$ Const на $\Sigma$ соответствуют окружсности $\sigma_{\rho}$ радиуса $f(\rho)$ и с иентром в $\mathbf{p}(\rho))$. Тогда поверхность $\Sigma$ является поверхностью вращения.

Заметим, что так как рассмотрения ведутся лишь в классе $C^{1}$, то мы не можем говорить об окружностях $\sigma_{\rho}$ как о линиях кривизны.

ДоКАЗАТЕЛЬСТво. Выпишем условия изометричности поверхности $\Sigma$ данной метрике $d s^{2}$. Получим уравнения:

$$
\begin{gathered}
\mathbf{p}^{\prime 2}+f^{\prime 2}+f \mathbf{e}_{\rho}^{\prime 2}+2\left(\mathbf{p}^{\prime} \mathbf{e}\right) f^{\prime}+2\left(\mathbf{p} \mathbf{e}_{\rho}^{\prime}\right) f=1 \\
\left(\mathbf{p}^{\prime} \mathbf{e}_{\varphi}^{\prime}\right)+\left(\mathbf{e}_{\rho}^{\prime} \mathbf{e}_{\varphi}^{\prime}\right) f=0 \\
f^{2} \mathbf{e}_{\varphi}^{\prime 2}=B^{2}
\end{gathered}
$$

Установим вид вектор-функции $\mathbf{e}(\rho, \varphi)$. Из соотношений $\left(\mathbf{q e}_{\varphi}^{\prime}\right)=0$ и $\left(\mathbf{e e}_{\varphi}^{\prime}\right)=0$ и (12) заключаем, что $\mathbf{e}_{\varphi}^{\prime}$ имеет представление вида $\mathbf{e}_{\varphi}=(\mathbf{e} \times \mathbf{q}) s(\rho)$, где $s(\rho)=$ $B(\rho) / f(\rho)$. Положим $\mathbf{e}=x \mathbf{i}+y \mathbf{j}+z \mathbf{k}, \mathbf{q}=\mathbf{i} q_{1}+\mathbf{j} q_{2}+\mathbf{k} q_{3}$ (где $\mathbf{i}, \mathbf{j}, \mathbf{k}-$ стандартный базис в $\mathbb{R}^{3}$ ) и решим систему линейных уравнений с постоянными (относительно $\varphi$ ) коэффициентами

$$
\begin{aligned}
x_{\varphi}^{\prime} & =\left(y q_{3}-z q_{2}\right) s(\rho), \\
y_{\varphi}^{\prime} & =\left(z q_{1}-x q_{3}\right) s(\rho), \\
z_{\varphi}^{\prime} & =\left(x q_{2}-y q_{1}\right) s(\rho) .
\end{aligned}
$$


Общее решение е этого уравнения, ортогональное к q, имеет вид (считаем, что $\left.q_{3} \neq 0\right)$ :

$$
\begin{aligned}
& c_{1}(\rho)\left\{\cos \psi,-\frac{q_{1}^{2}+q_{3}^{2}}{q_{1}^{2} q_{2}^{2}+q_{3}^{2}}\left(q_{1} q_{2} \cos \psi+q_{3} \sin \psi\right), \frac{q_{1}^{2}+q_{3}^{2}}{q_{1}^{2} q_{2}^{2}+q_{3}^{2}}\left(q_{2} \sin \psi-q_{1} q_{3} \cos \psi\right)\right\} \\
& +c_{2}(\rho)\left\{\sin \psi, \frac{q_{1}^{2}+q_{3}^{2}}{q_{1}^{2} q_{2}^{2}+q_{3}^{2}}\left(q_{3} \cos \psi-q_{1} q_{2} \sin \psi\right),-\frac{q_{1}^{2}+q_{3}^{2}}{q_{1}^{2} q_{2}^{2}+q_{3}^{2}}\left(q_{1} q_{3} \sin \psi+q_{2} \cos \psi\right\},\right.
\end{aligned}
$$

где положено $\psi=s \varphi$. Из условия единичности е получаем, что коэффициенты $c_{1}, c_{2}$ должны удовлетворять соотношению

$$
c_{1}^{2}+c_{2}^{2}=\frac{\left(q_{1}^{2} q_{2}^{2}+q_{3}^{2}\right)^{2}}{\left(1-q_{1}^{2}\right)\left(1-q_{2}^{2}\right)\left(q_{1}^{2}+q_{3}^{2}\right)} \equiv A^{2} .
$$

Представляя $c_{1}$ и $c_{2}$ в виде $c_{1}=A \sin \theta(\rho), c_{2}=A \cos \theta(\rho)$, где $\theta(\rho)$ - произвольная гладкая функция, для е получаем следуюшее представление

$$
\mathbf{e}=\mathbf{a}(\rho) \cos (\varphi s)+\mathbf{b}(\rho) \sin (\varphi s)
$$

где единичные и взаимно ортогональные векторы $\mathbf{a}(\rho), \mathbf{b}(\rho)$ в явном виде выражаются через компоненты вектора q и функцию $\theta(\rho)$. Для векторов $\mathbf{a}$ и $\mathbf{b}$ выполнены следующие соотношения

$$
\left(\mathbf{a a}^{\prime}\right)=0, \quad\left(\mathbf{b b}^{\prime}\right)=0, \quad\left(\mathbf{a b}^{\prime}\right)+\left(\mathbf{b} \mathbf{a}^{\prime}\right)=0
$$

Выгислив $\mathbf{e}_{\varphi}^{\prime}=-s \mathbf{a} \sin (\varphi s)+s \mathbf{b} \cos (\varphi s)$ и $\mathbf{e}_{\rho}^{\prime}=\mathbf{a}^{\prime} \cos (\varphi s)-\varphi s^{\prime} \mathbf{a} \sin (\varphi s)+$ $\mathbf{b}^{\prime} \sin (\varphi s)+\varphi s^{\prime} \mathbf{b} \cos (\varphi s)$ и подставив их значения сначала в (11), а затем в (10), с учетом (13) найдем соотношения $s^{\prime}=0$, т.е. $s(\rho)=$ const, $\left(\mathbf{a b}^{\prime}\right)=\left(\mathbf{b a}^{\prime}\right)=0$, $\left(\mathbf{a}^{\prime} \mathbf{b}^{\prime}\right)=0, \mathbf{a}^{\prime 2}=\mathbf{b}^{\prime 2}$, откуда получаем, что векторы $\mathbf{a}$ и $\mathbf{b}$-постоянные. Из этих же уравнений получаем равенства $\left(\mathbf{a p}^{\prime}\right)=\left(\mathbf{b p}^{\prime}\right)=0$, следовательно, линия центров с радиус-вектором $\mathbf{p}(\rho)$ имеет постоянное направление, т.е. она является прямой, причем ортогональной к векторам е, описывающим окружности. Тем самым мы установили, что поверхность $\Sigma$ действительно является поверхностью вращения.

ЗАмечАнИЕ. Обратим внимание, что у тора вращения тоже есть система окружностей с центрами вдоль кривой линии центров, однако если записать уравнение тора по отношению к метрике $d s^{2}=d \rho^{2}+B^{2}(\rho) d \varphi^{2}$, взяв за основу эту линию центров, то оно будет иметь вид $\mathbf{p}(\varphi)+f(\varphi) \mathbf{e}(\rho, \varphi)$, т.е. в этом представлении роли переменных $\rho$ и $\varphi$ другие.

5. Продолжим доказательство теоремы. Теперь мы предположим, что поверхность $S$ имеет объявленную в условиях теоремы гладкость класса $C^{2}$. Тогда мы в общем случае не можем гарантировать, что в качестве координатных линий можно взять два семейства линий кривизны. Покажем, что на самом деле в наших условиях на $S$ можно ввести координаты в линиях кривизны. Мы знаем (в предположении только, что $\left.S \in C^{2}\right)$, что на плоскости $(\xi, \eta)$ одно семейство линий кривизны определяется как линии уровня $R(\xi, \eta)=$ Const, причем на $S$ эти линии являются дугами окружностей. Следовательно, нам нужно показать, что на 
плоскости $(\xi, \eta)$ линии $R(\xi, \eta)=\beta$ и ортогональные к ним линии можно рассматривать как координатные линии новых переменных $(\alpha, \beta)$ с $C^{1}$-гладким переходом от $(\xi, \eta)$ к $(\alpha, \beta)$. Так как линии $R(\xi, \eta)=\beta$ на $S$ соответствует дуга окружности, которую можно считать как полученную сечением поверхности $S$ некоторой плоскостью, то каждая линия $R(\xi, \eta)=\beta$ на $(\xi, \eta)$-плоскости будет $C^{2}$-гладкой. Пусть $a(\beta) x+b(\beta) y+c(\beta) z=1-$ уравнения плоскостей, даюших в сечении с $S$ линии кривизны, вдоль которых главный радиус кривизны $R(\xi, \eta)=\beta$. Коэффициенты $a(\beta), b(\beta), c(\beta)$ этих плоскостей можно считать класса гладкости $C^{1}$. Действительно, пусть $M_{0} \in S$ - рассматриваемая точка, и $\sigma$ - линия кривизны на $S$, проходящая через $M_{0}$. Выберем на $\sigma$ три точки $M_{1}, M_{2}, M_{3}$. Так как $\sigma-$ дуга окружности, то эти точки не лежат на одной прямой. Выпустим из этих точек $M_{i}$ линии кривизны $\gamma_{i}$ второго семейства. Эти $C^{1}$-гладкие кривые имеют на плоскости $(\xi, \eta)$ уравнения вида $\xi=\xi_{i}(\beta), \eta=\eta_{i}(\beta), i=1,2,3$, и достаточно малые их дуги не пересекаются. Тогда $a(\beta), b(\beta), c(\beta)$ могут быть выражены в явном виде из формул для уравнения плоскости, проходяшей через три точки с радиус-векторами $\mathbf{r}\left(\xi_{i}(\beta), \eta_{i}(\beta)\right)$, и поэтому они действительно будут иметь гладкость класса $C^{1}$. Уравнение $a(\beta) x(\xi, \eta)+b(\beta) y(\xi, \eta)+c(\beta) z(\xi, \eta)=1$ определяет те же линии, что уравнение $R(\xi, \eta)=\beta$. Ортогональные к этим линиям кривые определяются как решения уравнения

$$
\left(a(\beta) x_{\eta}+b(\beta) y_{\eta}+c(\beta) z_{\eta}\right) d \xi-\left(a(\beta) x_{\xi}+b(\beta) y_{\xi}+c(\beta) z_{\xi}\right) d \eta=0
$$

Подставляя сюда вместо $\beta$ выражение $R(\xi, \eta)$, имеем, что семейство ортогональных траекторий определяется как решения обыкновенного дифференциального уравнения с правой частью класса $C^{1}$, следовательно, [4, с. 143] уравнения этого семейства траекторий можно представить в виде $f(\xi, \eta)=\alpha$, где $f(\xi, \eta) \in C^{1}$ первый интеграл уравнения. Так как кривые $f(\xi, \eta)=\alpha$ ортогональны к линиям $R(\xi, \eta)=\beta$, а координаты $(\xi, \eta)$ конформные, то на $S$ им соответствуют линии кривизны. Получается, что и в случае $S \in C^{2}$ на $S$ можно ввести координаты в линиях кривизны, и дальше можно повторить рассуждения как для случая $S \in C^{3}$, так как мы использовали наличие третьих производных только для обоснования возможности введения координат в линиях кривизны. Таким образом, кроме найденных раньше сферы и параболоида, мы новых поверхностей не получаем.

6. Итак, для случая $K \neq 0$ теорема доказана. Рассмотрим теперь случай $K \equiv 0$. Пусть в равенстве (3) равны нулю оба сомножителя, т.е. $K=0$ и $4+4 H h=0$. Следовательно, $H \neq 0$. Тогда имеем также уравнения (4), поэтому получаем, что $R=$ Const, $H=$ Const, $h=$ Const. Рассмотрим на $S$ некоторую линию кривизны $\sigma$, вдоль которой главный радиус кривизны $R=$ Const. Векторы $R \mathbf{n}$, исходящие из точек кривой $\sigma$, пересекаются в одной точке. Возьмем теперь произвольную образуюшую $l$, пересекаюшую $\sigma$. Эта образуюшая является линией кривизны 2-го семейства. Нормаль к $S$ вдоль $l$ постоянна, поэтому векторы $R \mathbf{n}$, исходящие из точек $l$, описывают некоторый отрезок прямой $\Gamma$, параллельной $l$. Так как прямая $\Gamma$ не зависит от выбора образуюшей $l$, то все образуюшие оказываются параллельными одной прямой $\Gamma$, следовательно, $S$ является цилиндрической поверхностью. Покажем, что $S$ - область на прямом круговом цилиндре. Так как 
метрика на $S$ локально евклидова, то можем считать, что в окрестности рассматриваемой точки метрика имеет вид $d s^{2}=d \xi^{2}+d \eta^{2}$. Тогда для радиус-вектора $\mathbf{r}(\xi, \eta)$ имеем уравнение $\Delta \mathbf{r}=2 H \mathbf{n}$, т.е. $\mathbf{r}$ удовлетворяет квазилинейному эллиптическому уравнению с аналитическим (постоянньм) коэффициентом $H$, поэтому $S$ является аналитической поверхностью. А тогда она допускает представление в так называемых асимптотических координатах (или, по-другому, с явным указанием образующих) вида

$$
\mathbf{r}(u, v)=\mathbf{t}(u)+v \mathbf{l}(u), \quad \mathbf{l}^{2}=1
$$

где направляющая $\mathbf{t}$ и образующие $\mathbf{l}$ аналитически зависят от $u$ [5] (если не ссылаться на аналитичность $S$, то согласно [6] для $S \in C^{2}$ в (14) можно гарантировать только $\mathbf{t} \in C^{2}, \mathbf{l} \in C^{1}$, что, впрочем, для нас было бы вполне достаточно, так как мы уже доказали, что 1 - постоянный единичный вектор). Далее, в (14) мы можем считать, во-первых, что $\mathbf{l}$ ортогональна к $\mathbf{t}^{\prime}$ (в противном случае достаточно сделать замену переменных $u=u, w=v-f(u)$, где $f(u)=-(\mathbf{l t}(u))$, и получится представление вида $\mathbf{r}(u, w)=\mathbf{T}(u)+w \mathbf{l}(u)$ с требуемым свойством), во-вторых, что $\mathbf{t}^{\prime 2}(u)=1$. Считаем, что постоянньй вектор 1 направлен по оси $z$ и направляющая кривая имеет радиус-вектор $\mathbf{t}=\{x, y, 0\}$. Тогда легко вычислить, что $L=-k, M=0, N=0,2 H=-2 k=$ Const, где $k-$ кривизна плоской направляющей, которая, таким образом, оказывается окружностью, что и утверждалось.

7. Осталось рассмотреть случай, когда (3) выполняется только за счет равенства $K=0$. Пусть рассматриваемая точка $M_{0} \in S$ не является точкой уплощения. Тогда в ее окрестности можно ввести систему координат $(u, v)$, в которой радиус-вектор $S$ имеет вид (14) с $\mathbf{t} \in C^{2}, \mathbf{l} \in C^{1}$ (см. [6]). Переход от $(\xi, \eta)$ к $(u, v)-$ класса $C^{1}$, поэтому $\mathbf{n}(u, v) \in C^{1}$. Вдоль каждой образующей $u=u_{0}=$ Const нормаль к $S$ постоянна, поэтому концы векторов $-h\left(u_{0}, v\right) \mathbf{n}\left(u_{0}, v\right)$ опишут плоскую линию $\Gamma \in S^{*}$, изометричную по построению $S^{*}$ отрезку образующей, на который линия Г ортогонально проектируется. А это возможно, если только линия Г сама является прямолинейным отрезком, параллельным образующей. Значит, $h\left(u_{0}, v\right)$ вдоль образующей постоянна, поэтому $h=h(u)$. Поверхность $S^{*}$ имеет радиус-вектор $\mathbf{r}^{*}(u, v)=\mathbf{t}(u)+v \mathbf{l}(u)-h(u) \mathbf{n}(u)$. Приравнивая метрики $d s^{2}$ и $d s^{* 2}$, получаем уравнения

$$
\begin{gathered}
h^{\prime 2}+h^{2} \mathbf{n}^{\prime 2}-2 h\left(\mathbf{t}^{\prime} \mathbf{n}^{\prime}\right)-2 v h\left(\mathbf{l}^{\prime} \mathbf{n}^{\prime}\right)=0 \\
h\left(\mathbf{l n}^{\prime}\right)=0 .
\end{gathered}
$$

Из (15) имеем, что коэффициент при $v$ равен нулю, т.е. $\left(\mathbf{l}^{\prime} \mathbf{n}^{\prime}\right)=0$ (так как считаем, что $h \neq 0)$. Следовательно, с учетом (16), $\mathbf{n}^{\prime} \perp \mathbf{l}, \mathbf{n}^{\prime} \perp \mathbf{l}^{\prime}$, поэтому если $\mathbf{1}^{\prime} \neq \mathbf{0}$, то $\mathbf{n}^{\prime}=\left(\mathbf{l} \times \mathbf{l}^{\prime}\right) s(u)$. Подставляя это значение $\mathbf{n}^{\prime}$ в $(15)$, получаем $h^{\prime 2}+h^{2} \mathbf{n}^{\prime 2}=0$, так как для развертываюшейся поверхности $\left(\mathbf{t}^{\prime} 1 \mathbf{l}^{\prime}\right)=0$. Следовательно, или $\mathbf{n}^{\prime 2}=0, h^{\prime 2}=0$, т.е. $S$ является плоской областью и изометрия состоит в сдвиге на $h=$ Const по нормали, или же $\mathbf{n}^{\prime 2} \neq 0$ и искомой изометрии нет. Остается рассмотреть случай $\mathbf{l}^{\prime}=0$, когда образуюшие параллельны одному направлению, т.е. когда $S$ является цилиндрической поверхностью. Направим ось $z$ параллельно образующим, а в качестве направляющей возьмем ортогональное 
к образующим сечение и параметризуем ее длиной дуги. Получим представление $S: \mathbf{t}(s)+v \mathbf{k},(\mathbf{t k})=0, \mathbf{t}^{\prime 2}=1, \mathbf{t}(s) \in C^{2}$. Пусть $\mathbf{t}(s)=\{x(s), y(s), z=0\}$. Тогда $\mathbf{t}^{\prime}=\left\{x^{\prime}, y^{\prime}, 0\right\}, \mathbf{n}=\left\{y^{\prime},-x^{\prime}, 0\right\}, \mathbf{n}^{\prime}=\left\{y^{\prime \prime},-x^{\prime \prime}, 0\right\}$ и уравнение (15) для $h$ принимает вид

$$
h^{\prime 2}+k^{2} h^{2}-2 h k=0
$$

где $k$ - кривизна направляюшей линии.

Для уравнения (17) имеем два возможных варианта решения. В первом варианте $h^{\prime} \equiv 0, h=2 / k=$ Const. В этом случае $S$ является прямым круговым цилиндром и $h=$ Const, т.е. имеем рассмотренный ранее случай. Во втором варианте $h$ определяется как общее решение дифференциального уравнения (17). Локально оно существует при любом начальном данном $h(0)=h_{0}$, удовлетворяюшем неравенству $2 h_{0} k(0)-h_{0}^{2} k^{2}(0)>0$, следовательно, любая цилиндическая поверхность $S \in C^{2}$ локально допускает изометрии рассматриваемого вида, которые при дополнительных условиях (а именно, если уравнение (17) имеет семейство решений $h(u, \varepsilon)$, непрерьвное по $\varepsilon$, с $h(u, 0)=0)$ являются изгибанием. Впрочем, примеры таких уравнений (17) нам неизвестны - легко показать, что решений вида $h(u, \varepsilon)=\varepsilon h_{1}(u)+\cdots$ уравнение $(17)$ не допускает, так что если изгибания есть, то зависимость их от $\varepsilon$ должна быть более сложной, без дифференцируемости по $\varepsilon$.

В случае $k=$ Const (т.е. направляющая цилиндра - окружность радиуса $R=1 / k)$ решением уравнения (17) являются две функции $h_{1}=R\left(1+\sin \left(k s+C_{1}\right)\right)$ и $h_{2}=R\left(1-\sin \left(k s+C_{2}\right)\right)$, которые имеют период $2 \pi R$ только при $C_{1}$ и $C_{2}$ целократных $2 \pi$, что соответствует начальному условию $h(0)=R$. При таких $C_{i}$ каждой функции $h_{i}$ по уравнению $\mathbf{r}+h_{i} \mathbf{n}$ соответствует дважды обходимая окружность соответственно с центром в $O_{i}$ (рис. 1 ) радиуса $R / 2$. Если обход данной окружности начинается в точке $A_{0}$, то после обхода полуокружности $A_{0} B A$ ветви для $h$ можно поменять, и ветвь для $h_{1}$ из $A_{0} B A$ можно продолжить как ветвь $h_{2}$ для $A C A_{0}$ и наоборот. Получаем, что для кругового цилиндра существуют 4 цилиндра с искомой изометрией: можно взять как изометричные поверхности круговые цилиндры с дважды обходимыми окружностями с центрами в $O_{1}$ и $O_{2}$ соответственно, или же цилиндр с направляющей в виде "восьмерки" (букет двух окружностей с центрами в $O_{1}$ и $O_{2}$ ) с двумя разными способами обхода - сначала обходится нижняя окружность, потом верхняя, или наоборот (при этом обход обеих окружностей проводится против часовой стрелки, так что в целом получается цилиндрическая поверхность с нарушением $C^{1}$-гладкости вдоль двух совпадающих в пространстве образующих).

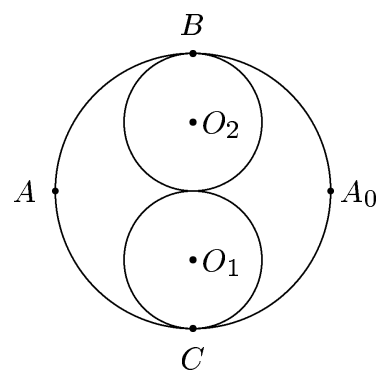

Рис. 1 
Для цилиндра с эллиптической направляющей картина сложнее; самое главное, что тогда уравнение (17) не имеет периодического решения. Во всяком случае, для конкретных эллипсов компьютерные вычисления, проведенные А. А. Тужилиным, показали, что решения уравнения (17) в зависимости от выбора начального условия $h(0)$ определяют семейство из незамкнутых $h$-кривых с довольно сложными взаимопереходами, не выходящих за область, полученную объединением эллипса и области внутри кривой $L$ с уравнением $\mathbf{r}+2 R \mathbf{n}$, где $R-$ радиус кривизны эллипса (см. рис. 2, где эллипс расположен с большой осью вдоль вертикальной оси, кривая $L$ имеет два самопересечения и она вычерчена жирной линией в горизонтальной вытянутости). Компьютерньй рисунок показывает, что эволюта эллипса является огибающей семейства $h$-кривых, что, впрочем, верно для любой кривой. Действительно, если в точке $s=s_{0}$ на исходной кривой взять начальное условие $h\left(s_{0}\right)=R=1 / k\left(s_{0}\right)$, то начальная точка соответствуюшей $h$-линии будет находиться на эволюте; кроме того, в этой точке касательньй вектор к эволюте $\mathbf{r}^{\prime}\left(s_{0}\right)+R^{\prime}\left(s_{0}\right) \mathbf{n}\left(s_{0}\right)+R \mathbf{n}^{\prime}\left(s_{0}\right)=R^{\prime}\left(s_{0}\right) \mathbf{n}\left(s_{0}\right)$ параллелен касательному вектору $\mathbf{r}^{\prime}\left(s_{0}\right)+h^{\prime}\left(s_{0}\right) \mathbf{n}\left(s_{0}\right)+h \mathbf{n}^{\prime}\left(s_{0}\right)=h^{\prime}\left(s_{0}\right) \mathbf{n}\left(s_{0}\right)$ к $h$-линии.

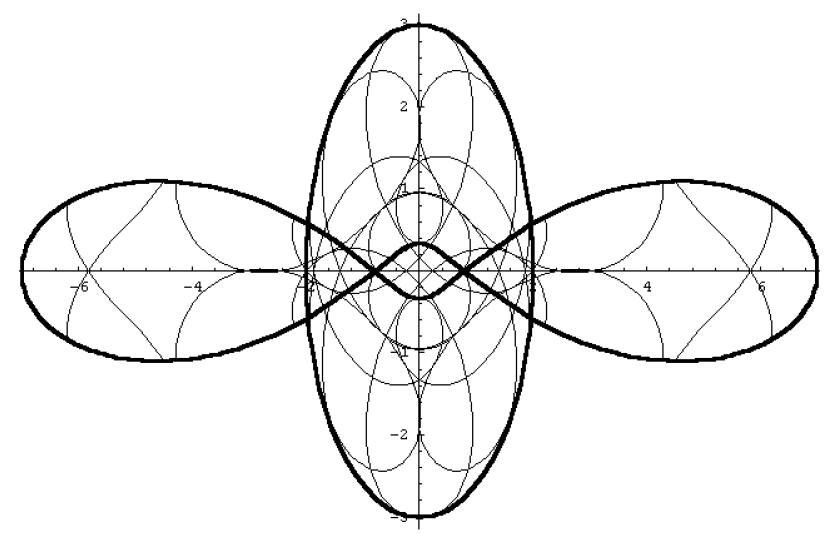

Рис. 2

Итак, теорема доказана, причем упоминаемый в теореме с двиг $h$ по нормали для цилиндрических поверхностей определяется решением дифференциального уравнения (17). Если бы удалось выделить классы замкнутых плоских кривых, для которых уравнение (17) имеет периодическое решение, то были бы описаны полные цилиндры, допускающие изометрии описьваемого вида на другие цилиндры с сохранением образующих.

8. В заключение рассмотрим вкратце общий случай, когда изометрии вида (1) порождают конформные отображения, которые априори не предполагаются тождественными, т.е. изометрия $S \rightarrow S^{*}$ не обязательно идет по нормали и, соответственно, не обязательно по равенству внутренних координат. Эта задача оказывается несравненно более трудной, и мы не имеем полного геометрического описания 
поверхностей, допускающих такие изометрии. Здесь, впрочем, в постановке самой задачи возможны существенные модификации и уточнения. Напомним, как появляется квазиконформное отображение $S \rightarrow S$, порожденное данной изометрией $S \rightarrow S^{*}$. Пусть точке $M \in S$ по этой изометрии соответствует точка $M^{*} \in S^{*}$. Спроектируем точку $M^{*}$ на $S$, т.е. опустим из $M^{*}$ нормаль к $S$. Обозначим эту операцию проектирования через $g$; пусть $g\left(M^{*}\right)=\widetilde{M} \in S$. Тогда, как показано в [1], отображение $f: \widetilde{M} \rightarrow M$ является квазиконформным отображением, удовлетворяющим некоторому уравнению Бельтрами. Следовательно, если поверхность $S$ открытая или с краем, то обратное отображение $f^{-1}: M \rightarrow \widetilde{M}$ в принципе может переводить $S$ в часть $S$ (но при этом нужно предполагать, что отображение $g: S^{*} \rightarrow S$ инъективно). Трудность здесь в том, что нам заранее не известна область $D=g\left(S^{*}\right)=f^{-1}(S) \subset S$, т.е. область определения квазиконформного (и, в частности, конформного) отображения $f: D \rightarrow S$ в общем случае заранее не задана, но образ отображения должен совпасть со всей рассматриваемой поверхностью $S$. Поэтому эту область или надо искать из каких-либо соображений (например, если поверхность компактна и изометричная ей поверхность $S^{*}$ достаточно близка к $S$, то $D=S$ ), или же надо ее задавать априори. В частности, если $S$ - поверхность с краем, то можем предположить, что край изометричной ей поверхности $S^{*}$ проектируется по нормали на край $S$. Тогда получим, что предполагаемое конформное отображение $f$ должно быть отображением $S$ на себя. Если мы предположим, что поверхность $S$ имеет достаточно сложную топологию, например, она гомеоморфна плоской области связности больше 2 или многообразию рода больше 0 и с краем, то конформные отображения таких областей на себя являются тождественными [7, с. 286], поэтому в этих случаях имеем описанную в теореме 1 ситуацию. Далее, если в других случаях мы предположим, что на $\partial S$ изометрия $S \rightarrow S^{*}$ идет по нормали, то соответствующее конформное отображение будет тождественным (для этого на самом деле достаточно, чтобы изометрия была по нормали только в трех точках края), и мы снова получим по теореме 1 полный ответ.

Для локального описания поверхностей, допускающих изометрии с соответствующим конформным отображением, мы предположим, что поверхность $S$ имеет гладкость класса $C^{3}$, а изометричная ей поверхность $S^{*}-$ класса $C^{2}$. Конфформность отображения, порождаемого изометрией $(1)$, означает, что метрика $d s^{*^{2}}$ имеет конформньй вид, т.е.

$$
\begin{gathered}
2 h L+\left(2 H L-K \lambda^{2}\right) h^{2}+h_{\xi}^{2}=2 h N+\left(2 H N-K \lambda^{2}\right) h^{2}+h_{\eta}^{2} \\
2 h M+2 H M h^{2}+h_{\xi} h_{\eta}=0 .
\end{gathered}
$$

Из этих уравнений мы можем найти $h$. Сначала находим $h_{\xi}$ и $h_{\eta}$. Имеем

$$
\begin{gathered}
h_{\xi}^{2}=A\left(h+H h^{2}\right), \quad A=N-L \pm \sqrt{(L-N)^{2}+4 M^{2}} \\
h_{\eta}^{2}=B\left(h+H h^{2}\right), \quad B=L-N \pm \sqrt{(L-N)^{2}+4 M^{2}} \\
h_{\xi} h_{\eta}=-2 M\left(h+H h^{2}\right) .
\end{gathered}
$$

Уравнения (18)-(20) позволяют выделить, прежде всего, следующие частные случаи. 
1) $h=0$; тогда $S^{*}$ обязана совпадать как множество точек с $S$, и если при этом имеем непрерывное семейство поверхностей $S_{\varepsilon}^{*}$, изометричных $S$, то получаем, что поверхность $S$ допускает изгибания скольжения, а тогда она имеет метрику поверхности вращения, локальный вариант см., например, в [8, с. 326 - 328], глобальное описание $S$ см. в [1] (открытый вопрос: пусть изометричная $S$ поверхность $S^{*}$ совпадает с $S$ как множество точек, но не получается из $S$ движением, и недискретность изометрии априори неизвестна, т.е. неизвестно, можно ли включить $S^{*}$ в семейство изгибаний; обязана ли $S$ иметь и в таком случае метрику врашения?). Очевидно, более подробную информацию о строении $S$, по крайней мере, в локальном варианте дать нельзя, так как любая реализация в $\mathbb{R}^{3}$ метрики врашения в области, ограниченной одной или двумя полными параллелями, допускает изгибания с $h=0$.

2) $1+h H=0$; тогда $h=$ Const $=-1 / H$. Следовательно, средняя кривизна поверхности постоянна и отлична от нуля. Здесь без учета возможного вида конформных отображений $z=f(\zeta), f: \bar{M}(\xi, \eta) \rightarrow M(x, y), z=x+i y, \zeta=\xi+i \eta$, можно лишш сказать, что должно быть выполнено равенство

$$
\lambda^{2}[z(\zeta)]=\lambda^{2}(\zeta)\left(1-\frac{K}{H^{2}}\right) /\left|\frac{\partial z}{\partial \zeta}\right|^{2} .
$$

Как видим, в условии (21) участвуют и внутренние, и внешние характеристики поверхности; каков их смысл или, точнее, каковы их последствия для описания возможного вида поверхностей, допускающих изометрии с соответствующими свойствами - нам неизвестно.

Наконец, рассмотрим основной случай, когда $h \neq$ Const. Сначала заметим, что направления $\left(h_{\xi}: h_{\eta}\right)$ и $\left(-h_{\eta}: h_{\xi}\right)$ удовлетворяют уравнению линий кривизны $-M d \xi^{2}+(L-N) d \xi d \eta+M d \eta^{2}=0$; следовательно, 2-е семейство линий кривизны состоит из линий уровня $h=$ Const. Это - обобщение и аналогия с тем, что мы имели в теореме 1. Дифференцируя уравнение (18) по $\eta$, а (19) - по $\xi$, мы из получившихся уравнений исключаем $h_{\xi \eta}$ и с учетом (20) приходим к уравнению для определения $h$ :

$$
h=-1 /\left(H+2 M \frac{2 M H_{\eta}+B H_{\xi}}{B A_{\eta}+2 M B_{\xi}}\right) .
$$

Поскольку $h$ однозначно определяется самой поверхностью, то изгибаний в рассматриваемом классе изометрий нет. Кроме того, дополнительно предполагая для $S$ гладкость класса $C^{4}$, вычислим из (22) производные $h$ по $\xi$ и $\eta$ и, подставив их в (18)-(20), получим условия, необходимые для существования рассматриваемых изометрий. Более подробного исследования допустимого вида таких поверхностей мы не проводили. Заметим только, что если соответствуюшее $h$ из (22) отображение $z=f(\zeta)$ (в обозначениях формулы (21)) можно включить в непрерывное семейство конформных отображений $z=f(\zeta, \varepsilon)$ при одном и том же $h$, то это означает, что $S^{*}$ допускает изгибания скольжения по себе. Следовательно, метрика поверхности $S^{*}$ и равная ей метрика $S$ являются метриками вращения. Вообще в этой теории поверхности $S$ и $S^{*}$ пока выступают как неравноправные; между тем, 
$S$ тоже можно проектировать ортогонально на $S^{*}$ и поэтому в дальнейших исследованиях стоило бы учесть эту симметрию $S$ и $S^{*}$.

Считаю своим приятным долгом выразить искреннюю благодарность А. А. Тужилину за интерес к этой работе и особенно за проведенное по его собственной инициативе компьютерное исследование уравнения, определяющего изометрии цилиндрической поверхности.

\section{Список литературы}

1. Сабитов И. Х. Квазиконформные отображения поверхности, порожденные ее изометрическими преобразованиями, и изгибания поверхности на себя // Фундамент. и прикл. матем. 1995. Т. 1. № 1. С. 281-288.

2. Сабитов И. Х. К вопросу о гладкости изометрий // Сиб. матем. журн. 1993. Т. 35. №4. C. $169-176$.

3. Hartman Ph., Wintner A. On the asymptotic curves of a surface // Amer. J. Math. 1951. V. 73. №1. P. $149-172$.

4. Хартман $\Phi$. Обыкновенные дифференциальнте уравнения. М.: Мир, 1970.

5. Ushakov V. Parametrization of developable surfaces by asymptotic lines // University of Melburn, Research Report. № 43, 1994. P. 1-9.

6. Hartman Ph., Nirenberg L. On spherical image maps whose Jacobians do not change sign // Amer. J. Math. 1959. V. 81. № 5. P. 901-920.

7. Неванлинна Р. Униформизация. М.: ИЛ, 1955.

8. Spivak M. A comprehensive introduction to Differential Geometry. V. 5. Berkley: Publish or Perish, 1979.

Московский государственншй Поступила в редакцию университет им. М.В. Ломоносова 31.01.1997 